\title{
Anderson Localization at Band Edges for Random Magnetic Fields
}

\author{
László Erdős;, David Hasler \\ Institute of Mathematics, University of Munich, \\ Theresienstr. 39, D-80333 Munich, Germany \\ lerdos@math.lmu.de, hasler@math.lmu.de
}

March 18, 2011

\begin{abstract}
We consider a magnetic Schrödinger operator in two dimensions. The magnetic field is given as the sum of a large and constant magnetic field and a random magnetic field. Moreover, we allow for an additional deterministic potential as well as a magnetic field which are both periodic. We show that the spectrum of this operator is contained in broadened bands around the Landau levels and that the edges of these bands consist of pure point spectrum with exponentially decaying eigenfunctions. The proof is based on a recent Wegner estimate obtained in [5] and a multiscale analysis.
\end{abstract}

\section{AMS Subject Classification: 82B44}

Running title: Magnetic localization at band edges

Key words: Anderson localization, random Schrödinger operator, magnetic Schrödinger operator

\section{Introduction}

The energy levels of a spinless quantum particle in the two dimensional Euclidean space $\mathbb{R}^{2}$ subject to a constant magnetic field $B_{0}$ are given by the Landau levels, $(2 n+1) B_{0}, n=0,1,2, \ldots$. A perturbation with an inhomogeneous random stationary magnetic field broadens the Landau levels into spectral bands. In this paper we prove Anderson localization near the band edges and we thus generalize our previous work [5] that treated only the bottom of the spectrum.

In the standard model for Anderson localization with a magnetic field (see, e.g., $[2,3,7,9,16]$ ) the random perturbation is given by an additive external potential. In our model the randomness is carried by the magnetic field. The main mathematical difference between these models is twofold.

First, the correlation structure of the local Hamiltonians for magnetic fields is much more involved. Assuming finite range correlations for the random perturbation, in case of potential perturbations the local Hamiltonians on distant domains are independent since the external potential acts locally. Some sufficiently decaying but not finite range correlations can also be treated with the known methods, see [4, 11] and references therein. In case of magnetic perturbations, it is the vector potential $A$ and not the magnetic field $B=\nabla \times A$ that appears directly in the Hamiltonian. Since the dependence of the vector potential on the magnetic field is nonlocal, the local Hamiltonians with distant domains are typically strongly correlated even for magnetic fields with a short range correlation. This strong correlation cannot be directly tackled with the standard methods of multiscale analysis, but using appropriate gauge transformations helps.

The second difference between random external potentials and random magnetic fields is that the energy depends monotonically on the external potential but not on the magnetic field. A cornerstone of any existing

\footnotetext{
*Partially supported by SFB-TR12 of the German Science Foundation
} 
proof of Anderson localization is the Wegner estimate whose standard proofs rely on monotonicity. Prior to our work [5], Wegner estimate, and hence localization, has only been proven for random magnetic fields with a zero flux condition [13] and for fields generated by stationary vector potentials in $[15,8]$, motivated by a method in [10]. Note that stationary vector potentials imply that the flux is zero on average.

In [5] we developed a new method to prove Wegner estimate for stationary random fields, i.e. for a model without monotonicity. In particular, zero-flux condition was not needed. We also proved Lifshitz tail at the bottom of the spectrum. These ingredients, combined with the usual multi-scale argument yielded localization at the bottom of the spectrum. In [6] we solved the same problem for the lattice model.

In the current paper we extend our method for higher band edges. The Wegner estimate and the multi-scale argument remain essentially unchanged and we will just quote the necessary results. The new ingredients are (i) the precise location of the higher band edges and (ii) an estimate on the Lifshitz tail. Both results are especially effective if the background constant field is strong compared with the random perturbation.

\section{Model and Statement of Results}

We work in $\mathbb{R}^{2}$ and we set $|x|_{\infty}:=\max \left\{\left|x_{1}\right|,\left|x_{2}\right|\right\}$ for any $x \in \mathbb{R}^{2}$. We shall denote magnetic fields by $B$. Let $A$ be a magnetic vector potential such that $\nabla \times A=B$. By $H(A)$ we denote the magnetic Schrödinger operator on $L^{2}\left(\mathbb{R}^{2}\right)$ with a bounded external potential $V$, i.e.,

$$
H(A)=(p-A)^{2}+V .
$$

We realize this as a self adjoint operator by means of the Friedrichs extension. If we refer to statements which are independent of the particular choice of gauge, with a slight abuse of notation, we shall occasionally write $H(B)$. In particular, we denote by $\sigma(H(A))$ the spectrum of the magnetic Schrödinger operator $H(A)$. Since the spectrum is gauge invariant sometimes we will also use the notation $\sigma(H(B))$.

We consider a deterministic magnetic field $B_{\operatorname{det}}(x)=B_{0}+B_{\mathrm{var}}(x)$ where $B_{0}$ denotes a constant magnetic field and $B_{\text {var }}$ is a perturbation that typically varies in space. We perturb this deterministic magnetic field by a random one, i.e., we consider

$$
B=B_{\omega}=B_{\mathrm{det}}+\mu B_{\mathrm{ran}}^{\omega}
$$

where $\mu \in(0,1]$ denotes the coupling constant and $B_{\text {ran }}^{\omega}$ is a random magnetic field constructed as follows.

We choose a profile function $u \in C_{0}^{1}\left(\mathbb{R}^{2}\right), 0 \leq u \leq 1$. Fix $k \in \mathbb{N}$ and define the lattice $\Lambda^{(k)}=\left(2^{-k} \mathbb{Z}\right)^{2}$. For $z \in \Lambda^{(k)}$ define

$$
\beta_{z}^{(k)}(x):=u\left(2^{k}(x-z)\right)
$$

The randomness is represented by a collection of independent random variables

$$
\omega=\left\{\omega_{z}^{(k)}: k \in \mathbb{N}, z \in \Lambda^{(k)}\right\} .
$$

We assume that all $\omega_{z}^{(k)}$ have zero expectation, and they satisfy a bound that is uniform in $z$

$$
\left|\omega_{z}^{(k)}\right| \leq \sigma^{(k)}:=C_{\mathrm{ran}} e^{-\rho k},
$$

with some $\rho>0$. By $v_{z}^{(k)}$ we shall denote the density function of $\omega_{z}^{(k)}$ (which strictly speaking might be a distribution). For each $(k, z) \in \mathcal{L}:=\bigcup_{k \in \mathbb{N}}\{k\} \times \Lambda^{(k)}$ we have a probability measure with density $v_{z}^{(k)}$. The associated product measure, $\mathbb{P}$, is probability measure on $\Omega=\mathbb{R}^{\mathcal{L}}$, and we denote expectation with respect to this measure by $\mathbb{E}$. We define the random magnetic field as

$$
B_{\mathrm{ran}}^{\omega}(x)=B_{\mathrm{ran}}(x):=\sum_{k=0}^{\infty} B^{(k)}(x), \quad B^{(k)}(x):=\sum_{z \in \Lambda^{(k)}} B_{z}^{(k)}(x), \quad B_{z}^{(k)}(x):=\omega_{z}^{(k)} \beta_{z}^{(k)}(x),
$$

i.e. $B_{\mathrm{ran}}^{\omega}$ is the sum of independent local magnetic fields on each scale $k$ and at every $z \in \Lambda^{(k)}$. 
The random magnetic field just constructed will in general live on infinitely many scales. This structure will be necessary to prove the Wegner estimate and hence the Anderson localization. Before that, we will state several results about the location of the spectrum. In these results the genuine multi-scale structure is not necessary; it is allowed that there is only one scale. This case is included in the above construction by choosing all $\omega_{z}^{(k)}=0$ for $k \geq 1$ (which corresponds to the case where the corresponding distribution is a point measure at the origin).

To state our results about the deterministic spectrum we need that the random magnetic field is stationary. This is ensured if we make the following assumption.

(i.i.d.) For any fixed $k \in \mathbb{N}$ the random variables $\left\{\omega_{z}^{(k)}: z \in \Lambda^{(k)}\right\}$ are identically distributed.

Theorem 2.1 [5, Theorem 3.2] Suppose $B_{\omega}$ is a random magnetic field a random magnetic field constructed in (2.1), (2.2), and (2.4), satisfying (2.3) and (i.i.d.). Assume $B_{\mathrm{var}}$ and $V$ are $\mathbb{Z}^{2}$-periodic. Then there exists a set $\Sigma \subset \mathbb{R}$ and a set $\Omega_{1} \subset \Omega$ with $\mathbb{P}\left(\Omega_{1}\right)=1$ such that for all $\omega \in \Omega_{1}$

$$
\sigma\left(H\left(B_{\omega}\right)\right)=\Sigma
$$

Henceforth we will denote by $\Sigma$ the almost sure deterministic spectrum of $H\left(B_{\omega}\right)$. The next two theorems provide estimates on the location of the deterministic spectrum. We define two specific configurations of the collection of random variables,

$$
\left(\omega_{+}\right)_{z}^{(k)}:=\operatorname{ess} \sup \left[\omega_{z}^{(k)}\right]_{+}, \quad\left(\omega_{-}\right)_{z}^{(k)}:=-\operatorname{ess} \sup \left[-\omega_{z}^{(k)}\right]_{+},
$$

where $[f]_{+}=\max (0, f)$ denotes the positive part. The configuration $\omega_{ \pm}$corresponds to maximal respectively minimal magnetic field. These configurations give rise to the following points close to the band edges of the deterministic spectrum (provided one has band structure),

$$
E_{n}^{-}:=\inf _{x \in \mathbb{R}^{2}}\left[(2 n+1) B_{\omega_{-}}(x)+V(x)\right], \quad E_{n}^{+}:=\sup _{x \in \mathbb{R}^{2}}\left[(2 n+1) B_{\omega_{+}}(x)+V(x)\right] .
$$

Moreover, we need that the derivatives of the fluctuations are not to large. This is quantified in terms of the following constants

$$
\begin{aligned}
& K_{2}^{ \pm}:=\left\|\nabla B_{\omega_{ \pm}}\right\|_{\infty}+\|\nabla V\|_{\infty}, \\
& K_{3}^{ \pm}=\left\|\nabla B_{\omega_{ \pm}}\right\|_{\infty}^{2} .
\end{aligned}
$$

Note that using (2.3) and the support properties of the profile function $u$ for any $\omega$ in the support of $\mathbb{P}$ we have

$$
\left\|\nabla B_{\text {ran }}^{\omega}\right\|_{\infty} \leq C \sum_{k} e^{-\rho k} 2^{k}\|\nabla u\|_{\infty} \leq\left(1-2 e^{-\rho}\right)^{-1}\|\nabla u\|_{\infty}
$$

i.e. a sufficient condition for $B_{\text {ran }}^{\omega}$ to be differentiable is $\rho>\ln 2$.

We will use the following theorem in the proof of our localization result to show that the interval for which we prove pure point spectrum, does in fact contain spectrum, (2.23) in Theorem 2.5.

Theorem 2.2 Suppose $B_{\omega}$ is a random magnetic field constructed in (2.1), (2.2), and (2.4), satisfying (2.3), (i.i.d.), and $0<b_{0} \leq B_{\omega_{ \pm}}$with some positive constant $b_{0}$. Assume $B_{\mathrm{var}}$ and $V$ are $\mathbb{Z}^{2}$-periodic. Then there exists a $C_{\mathrm{int}, n}$ such that

$$
\operatorname{dist}\left(\Sigma, E_{n}^{ \pm}\right) \leq C_{\mathrm{int}, n}\left[K_{2}^{ \pm} b_{0}^{-1 / 2}+K_{3}^{ \pm} b_{0}^{-2}\right] .
$$

The following theorem can be used to establish that the random Hamiltonian exhibits band structure under the additional condition that $B_{\mathrm{det}}=B_{0}$ is constant, i.e. $B_{\mathrm{var}}=0$ and $V=0$. 
Theorem 2.3 Let $B_{\mathrm{det}}=B_{0}$ be constant and $V=0$. Suppose $B_{\omega}$ is a random magnetic field constructed in (2.1), (2.2), and (2.4), satisfying (2.3), (i.i.d.), and $0<b_{0} \leq B_{\omega} \leq K_{0} b_{0}$ for some $K_{0}>1$. Assume that the essential support of $v_{z}^{(k)}$ is an interval (possibly consisting of a single point). Then there exist intervals $\Sigma_{n}=\left[\Sigma_{n}^{-}, \Sigma_{n}^{+}\right]$containing $B_{0}(2 n+1)$ such that

$$
\Sigma=\bigcup_{n=0}^{\infty} \Sigma_{n}
$$

There exists a constant $C_{n}$ (depending only on $n$ and $K_{0}$ ) such that

$$
\operatorname{dist}\left(\Sigma_{n}^{ \pm}, E_{n}^{ \pm}\right) \leq C_{n}\left[K_{2}^{ \pm} b_{0}^{-1 / 2}+K_{3}^{ \pm} b_{0}^{-2}\right] .
$$

Remark 2.4 Theorem 2.3 can be used to show that the spectrum exhibits band structure. For fixed $n$ and bounded random field $\left|B_{\mathrm{ran}}\right| \leq C$ the intervals $\Sigma_{n}$ will be disjoint for large $b_{0}$. However, the distance between neighboring Landau levels does not depend on $n$, but the width of $\Sigma_{n}$ is typically increasing in $n$. Thus for fixed $b_{0}$ and large $n$ the intervals $\Sigma_{n}$ will no longer be disjoint and the gaps close, i.e. the high-energy spectrum will be a half-life.

Below we list additional assumptions which are needed in [5] to show the Wegner estimate, and which are hence needed to prove localization. It is here where we need that the random magnetic field lives on arbitrarily small scales. The profile function satisfies one of the following two conditions for some sufficiently small $\delta$ : either

$$
u(x) \equiv 0 \quad \text { for } \quad|x|_{\infty} \geq \frac{1}{2}+\delta \quad \text { and } \quad u(x) \equiv 1, \quad \text { for } \quad|x|_{\infty} \leq \frac{1}{2}-\delta
$$

or

$$
u(x)=\delta^{2} u_{0}(x \delta) \quad \text { with some } u_{0} \in C_{0}^{1}\left(\mathbb{R}^{2}\right), \quad \int_{\mathbb{R}^{2}} u_{0}=1, \quad u_{0}(x) \equiv 0 \text { for }|x|_{\infty} \geq 1 .
$$

In both cases $\delta$ can be chosen as a sufficiently small positive number $\delta \leq \delta_{0} \leq 1$, and the threshold $\delta_{0}$ can be chosen as

$$
\begin{aligned}
\delta_{0} & =\frac{1}{3200} \quad \text { under condition (2.11) } \\
\delta_{0} & =\frac{1}{640+32\left\|\nabla u_{0}\right\|_{\infty}^{2}} \quad \text { under condition (2.12). }
\end{aligned}
$$

The density function $v_{z}^{(k)}$ is in $C_{0}^{2}(\mathbb{R})$ and satisfies

$$
\int_{\mathbb{R}}\left|\frac{\mathrm{d}^{2} v_{z}^{(k)}}{\mathrm{d} s^{2}}(s)\right| \mathrm{d} s \leq C\left[\sigma^{(k)}\right]^{-2}=C e^{2 \rho k},
$$

in particular the magnetic field has a non-trivial random component on every scale. The profile function satisfies

$$
U(x):=\sum_{z \in \mathbb{Z}^{2}} u(x-z) \geq c_{u} \quad \text { and } \sup _{x \in \mathbb{R}^{2}} U(x)=1,
$$

for some positive constant $c_{u}>0$. We are given positive numbers $b_{0}$ and $K_{0}>3$, such that

$$
0<2 b_{0} \leq B_{\operatorname{det}}(x) \leq\left(K_{0}-1\right) b_{0},
$$

Moreover, we assume that

$$
\sum_{k=0}^{\infty} \sigma^{(k)} \leq b_{0}
$$


i.e. $\left(1-e^{-\rho}\right) b_{0} \geq C_{\mathrm{ran}}$. It follows that

$$
0<b_{0} \leq B_{\omega} \leq K_{0} b_{0}
$$

We also assume the following condition on the potential

$$
\|V\|_{\infty} \leq b_{0} / 4 .
$$

The following list summarizes the assumptions for which a Wegner estimate was shown in [5] (see Theorem 5.1 below for the precise statement).

(W) $B_{\omega}$ is a random magnetic field constructed in (2.1), (2.2), and (2.4), satisfying (2.3), (2.11) or (2.12) for some $0<\delta \leq \delta_{0}$, and (2.14)-(2.17) hold with some parameters $K_{0}>3, b_{0}>0$ and $\rho>\ln 2$. Let also (2.19) hold.

Finally, we now collect the additional assumptions needed to prove the result about localization. Let $\tau>0$ be a parameter.

$\left(\mathbf{A}_{\boldsymbol{\tau}}\right)$ Hypothesis $(\mathbf{W})$ and (i.i.d.) hold, and $B_{\text {var }}$ and $V$ are $\mathbb{Z}^{2}$-periodic. The density function $v^{(0)}=v_{z}^{(0)}$ is supported on an interval and there exists a polynomial bound on its lower tail, in the sense that there exist constants $c_{v}>0$ such that for all $h \geq 0$ the probability distribution functions

$$
\nu_{+}(h):=\mathbb{P}\left(\omega^{(0)} \leq \omega_{+}^{(0)}-h\right), \quad \nu_{-}(h):=\mathbb{P}\left(\omega^{(0)} \geq \omega_{-}^{(0)}+h\right)
$$

satisfy

$$
\nu_{ \pm}(h) \leq c_{v} h^{\tau}
$$

To state the result we introduce the following quantity

$$
K_{2}:=\operatorname{ess} \sup _{\omega}\left\|\nabla B_{\omega}\right\|_{\infty}+\|\nabla V\|_{\infty} .
$$

Observe that one can derive an explicit upper bound on this constant with the help of (2.9).

Theorem 2.5 Let $\left(\mathbf{A}_{\tau}\right)$ hold for some $\tau>2$ and let $K_{0}, b_{0}$ be the parameters in $(\mathbf{W})$. Let $B_{\omega}=B_{\operatorname{det}}+$ $\mu B_{\mathrm{ran}}^{\omega}$ with $\mu \in(0,1]$ be the random magnetic field with a vector potential $A_{\omega}$. For every $n \in \mathbb{N}$ there exists an $\varepsilon_{n}>0$ and $U_{n}>0$ (independent of $b_{0}$ but depending on $K_{0}, \tau, \rho, \delta, C_{\mathrm{ran}}, c_{u}, c_{v}$ ) such that for any $b_{0}$ with $b_{0} \geq U_{n}\left(K_{2}^{4}+1\right)$ the interval

$$
I_{n}:=\left[E_{n}^{+}-\varepsilon_{n}, E_{n+1}^{-}+\varepsilon_{n}\right]
$$

is non-emtpy and for almost every $\omega$ the operator $H\left(A_{\omega}\right)$ has in $I_{n}$ pure point spectrum with exponentially decaying eigenfunctions. Moreover, this interval contains spectrum at least near its edges, i.e.

$$
\left(E_{n}^{+}-\varepsilon_{n}, E_{n}^{+}+\varepsilon_{n}\right) \cap \Sigma \neq \emptyset, \quad\left(E_{n+1}^{-}-\varepsilon_{n}, E_{n+1}^{-}+\varepsilon_{n}\right) \cap \Sigma \neq \emptyset .
$$

Remark 2.6 If $B_{\mathrm{var}}=V=0$, then it is a trivial consequence of Theorem 2.3 that both intersections (2.23) contain in fact an interval of nonzero length.

Remark 2.7 For a typical random magnetic field we have $E_{n+1}^{-}<E_{n}^{+}$for any sufficiently large $n$ (the lower threshold depends on $b_{0}$ ). This is the signature that the broadening of the Landau levels will overlap, see Remark 2.4.

Remark 2.8 We note that the assumption (i.i.d.) could be relaxed to the weaker assumption that for any fixed $k \in \mathbb{N}$ and all $z \in \Lambda^{(k)},\left\{\omega_{z}^{(k)}: z^{\prime}=z+w, w \in \mathbb{Z}^{2}\right\}$ are identically distributed. Our proofs show that the results of Theorems 2.3, 2.3, and 2.5 still hold under this weaker assumption. 
We will use the notation that $\mathbf{1}_{S}$ as well as $\chi_{S}$ denotes the characteristic function of a set $S$.

Let us now outline the remaining part of the paper. In Section 3, we prove a result, stated in Theorem 3.2 , which gives an outer bound on the spectrum of a magnetic Hamiltonian. It will be used in the proof of both Theorem 2.3 and Theorem 2.5. The result might be of interest of its own.

In Section 4, we prove Theorems 2.2 and 2.3. Theorem 2.2 will follow by choosing a suitable trial state. To prove Theorem 2.3 we will use the outer bound on the spectrum and a perturbation theory argument in the continuous spectrum.

Section 5 is devoted to the proof of Theorem 2.5. It will be based on the Wegner estimate shown in [5] and a multi-scale analysis as used in [5] to prove localization at the bottom of the spectrum. To this end, one needs an initial length scale estimate. In [5] an elementary lower bound on the spectrum was sufficient to obtain an initial length scale estimate for the bottom of the spectrum. This bound is not helpful for higher band edges and instead we use Theorem 3.2, which provides an outer bound on the spectrum. There is an additional difficulty originating from the fact that for the finite volume Hamiltonians there is always spectrum not only near the unperturbed Landau levels but also well in between them which corresponds to states which live close to the boundary. To deal with this difficulty, we use the Wegner estimate to estimate the exponential decay of the finite volume Hamiltonian in terms of the exponential decay of the infinite volume Hamiltonian, this is outlined in Subsection 5.1. In Subsection 5.2 we show the initial length scale estimate and in Subsection 5.3 we introduce the multi-scale analysis, which is used to prove Theorem 2.5.

\section{Outer bound on the spectrum}

Let $B(x)=B_{0}+B_{1}(x)$, where $B_{0}$ is a constant magnetic field and $B_{1}$ denotes a non-constant perturbation. We define

$$
e_{n, \min }[B]:=\inf _{x \in \mathbb{R}^{2}}[(2 n+1) B(x)+V(x)], \quad e_{n, \max }[B]:=\sup _{x \in \mathbb{R}^{2}}[(2 n+1) B(x)+V(x)] .
$$

These numbers correspond to the maximal broadening of the Landau levels $(2 n+1) B_{0}$ for the constant $B_{0}$ field if the perturbation given by $B_{1}$ and $V$ were considered by classical mechanics.

Remark 3.1 Note that

$$
E_{n}^{+}=e_{n, \max }\left[B_{\omega_{+}}\right], \quad E_{n}^{-}=e_{n, \min }\left[B_{\omega_{-}}\right] .
$$

To formulate the next theorem, we introduce the following quantities,

$$
\begin{aligned}
\hat{K}_{2} & :=\left\|\nabla B_{1}\right\|_{\infty}+\|\nabla V\|_{\infty} \\
\hat{K}_{3} & :=\left\|\nabla B_{1}\right\|_{\infty}^{2} .
\end{aligned}
$$

The following theorem shows that in the large $B_{0}$ regime the classical edges $e_{n, \min }[B]$ and $e_{n, \max }[B]$ give an outer bound on the true spectrum up to a small correction.

Theorem 3.2 Let $C_{1}>0$ and assume that

$$
C_{1}^{-1} B_{0} \leq B \leq C_{1} B_{0}
$$

Then for every $n \in \mathbb{N}$ there exists a constant $C_{\mathrm{ext}, n} \geq 0$ (depending only on $C_{1}$ ) such that the intervals $\hat{I}_{n}[B]:=\left(\hat{I}_{n}^{+}[B], \hat{I}_{n+1}^{-}[B]\right)$ with

$$
\begin{aligned}
\hat{I}_{n}^{+}[B] & :=e_{n, \max }[B]+C_{\text {ext }, n}\left[\hat{K}_{2} B_{0}^{-1 / 2}+\hat{K}_{3} B_{0}^{-2}\right], \\
\hat{I}_{n+1}^{-}[B] & :=e_{n+1, \min }[B]-C_{\text {ext }, n+1}\left[\hat{K}_{2} B_{0}^{-1 / 2}+\hat{K}_{3} B_{0}^{-2}\right],
\end{aligned}
$$

are in the resolvent set of $H(B)$. 
To prove the theorem we localize the resolvent in position space and treat it as a perturbation of the resolvent of a Hamiltonian with a constant magnetic field. The choice of the constant magnetic field will depend on specific location in position space.

Proof. We fix a gauge $A$ such that $\nabla \times A=B$. To localize in position space, we choose a profile function, which equals the normalized characteristic function of a disk, centered at the origin, with radius one, $g(x):=\frac{1}{\pi} \mathbf{1}_{\{|x|<1\}}$. We use this function to define a rescaled and translated version thereof, by setting $g_{u}(x):=g_{u, B_{0}}(x):=B_{0} g\left(B_{0}^{1 / 2}(x-u)\right)$, with $u \in \mathbb{R}^{2}$. The prefactor $B_{0}$ is chosen such that

$$
\int g_{u}(x) d u=1
$$

Moreover, we will denote the characteristic function of the support of $g_{u}$ by $\chi_{u}:=\chi_{u, B_{0}}:=\mathbf{1}_{\operatorname{supp} g_{u}}$.

Next we introduce suitable gauges to control the magnetic field on the support of $g_{u}$. For each $u \in \mathbb{R}^{2}$ we set

$$
B_{u, 0}:=B(u), \quad \text { and } \quad \delta_{u}[B]:=B-B_{u, 0},
$$

and we define the vector potentials

$$
\begin{aligned}
\widetilde{A}_{u, 0}\left(x_{1}, x_{2}\right) & :=\left(-\frac{B_{u, 0}}{2}\left(x_{2}-u_{2}\right), \frac{B_{u, 0}}{2}\left(x_{1}-u_{1}\right)\right) \\
\delta_{u}[\widetilde{A}]\left(x_{1}, x_{2}\right) & :=\left(-\frac{1}{2} \int_{u_{2}}^{x_{2}} \delta_{u}[B]\left(x_{1}, \xi\right) d \xi, \quad \frac{1}{2} \int_{u_{1}}^{x_{1}} \delta_{u}[B]\left(\xi, x_{2}\right) d \xi\right) \\
\widetilde{A}_{u} & :=\widetilde{A}_{u, 0}+\delta_{u}[\widetilde{A}] .
\end{aligned}
$$

As a consequence of the definition we have $\nabla \times \widetilde{A}_{u}=B$. Thus if we define the function

$$
\lambda_{u}(x)=\int_{\gamma_{x}}\left(\widetilde{A}_{u}-A\right) d \gamma_{x}
$$

where $\gamma_{x}$ denotes any differentiable path connecting the origin with $x \in \mathbb{R}^{2}$ (for example a straight line), then

$$
A=\widetilde{A}_{u}-\nabla \lambda_{u}
$$

The function $\lambda_{u}$ will be used below in (3.12) for a gauge transformation, and we set

$$
A_{u, 0}:=\widetilde{A}_{u, 0}-\nabla \lambda_{u} .
$$

As an immediate consequence of the definition we have

$$
\nabla \times A_{u, 0}=B_{u, 0} .
$$

Moreover, we introduce the notation $V_{u}=V(u), \delta_{u}[V]=V-V_{u}$, and for any vector potential $a$ we write $H_{u}(a)=(p-a)^{2}+V_{u}$.

We consider the following resolvent identity which depends on the position $u$,

$$
\frac{1}{z-H(A)} g_{u}=\frac{1}{z-H_{u}\left(A_{u, 0}\right)} g_{u}+\frac{1}{z-H(A)}\left[H(A)-H_{u}\left(A_{u, 0}\right)\right] \frac{1}{z-H_{u}\left(A_{u, 0}\right)} g_{u}
$$

which we integrate over $u$ as a weak integral and obtain, using the above gauge transformation,

$$
\frac{1}{z-H(A)}=\int \frac{1}{z-H_{u}\left(A_{u, 0}\right)} g_{u} d u+\frac{1}{z-H(A)} \int e^{-i \lambda_{u}} S_{u, z} e^{i \lambda_{u}} g_{u} d u
$$


where we defined

$$
S_{u, z}:=\left[H\left(\widetilde{A}_{u}\right)-H_{u}\left(\widetilde{A}_{u, 0}\right)\right] \frac{1}{z-H_{u}\left(\widetilde{A}_{u, 0}\right)} \chi_{u} .
$$

Now by (3.12) and the triangle inequality

$$
\left\|(z-H(A))^{-1}\right\| \leq\left\|\int \frac{1}{z-H_{u}\left(A_{u, 0}\right)} g_{u} d u\right\|+K\left\|(z-H(A))^{-1}\right\|,
$$

where

$$
K:=\left\|\int e^{-i \lambda_{u}} S_{u, z} e^{i \lambda_{u}} g_{u} d u\right\| .
$$

By Proposition 3.4, below, the first term on the right hand side of (3.14) is bounded. Thus $(z-H(A))^{-1}$ will be bounded and the theorem will follow, provided we show that $K<1$. But this follows from Lemma 3.3 and Lemma 3.5 provided $C_{\text {ext }, n}$ is chosen sufficiently large.

Lemma 3.3 For $u \in \mathbb{R}^{2}$ let $T_{u}$ be an operator in $L^{2}\left(\mathbb{R}^{2}\right)$ with integral kernel $T_{u}(x, y)$ depending measurably on $u, x, y$. Let $C$ be a bounded operator with integral kernel satisfying $\left|T_{u}(x, y)\right| \leq C(x, y)$ for all $u \in \mathbb{R}^{2}$. Then

$$
\left\|\int T_{u} g_{u} d u\right\| \leq\|C\| .
$$

Proof. Let $\psi \in L^{2}\left(\mathbb{R}^{2}\right)$. Then using (3.6) we find

$$
\left|\left[\int T_{u} g_{u} d u \psi\right](x)\right| \leq \int C(x, y) g_{u}(y)|\psi(y)| d y d u=[C|\psi|](x) .
$$

Thus

$$
\left\|\int T_{u} g_{u} d u \psi\right\| \leq\|C|\psi|\| \leq\|C\|\|\psi\|
$$

Proposition 3.4 Let $z \in \mathbb{C}$ with $\operatorname{dist}\left(\operatorname{Re} z, \bigcup_{n \in \mathbb{N}_{0}}\left[e_{n, \min }[B], e_{n, \max }[B]\right]\right)>0$ and $|\operatorname{Im} z| \leq B_{0}$. Suppose (3.3) holds. Then

$$
\int d u \frac{1}{z-H_{u}\left(A_{u, 0}\right)} g_{u}
$$

is bounded.

Proof. Recall that $A_{u, 0}$ is a vector potential for the constant magnetic field $B_{u, 0},(3.11)$. Thus by definition (3.1) it follows that $\sigma\left(H_{u}\left(A_{u, 0}\right)\right) \subset \bigcup_{n \in \mathbb{N}_{0}}\left[e_{n, \min }[B], e_{n, \max }[B]\right]$. Now we use Lemma A.1 in the appendix, to estimate the integral kernel of the resolvent. We conclude using (A.3), (A.4), and (3.3) that there exist an operator $D_{z}$ with integral kernel $D_{z}(x, y)$ such that for all $u \in \mathbb{R}^{2}$

$$
\left|\frac{1}{z-H_{u}\left(A_{u, 0}\right)}(x, y)\right| \leq D_{z}(x, y)
$$

with

$$
D_{z}(x, y)=\mathcal{D}_{z}(x-y), \quad \mathcal{D}_{z}(x) \leq C_{z}(1+\ln |x|) \exp \left(-c_{z}|x|^{2}\right),
$$

for some constants $C_{z}$ and $c_{z}>0$. From this it follows that $D_{z}$ is a bounded operator (with bound depending on $B_{0}$ ), since $\left\|\mathcal{D}_{z}\right\|_{1}$ is bounded and hence also the Fourier transform of $\mathcal{D}_{z}$. Now the claim follows in view of Lemma 3.3.

The next lemma estimates the operator $S_{u, z}$ for $z$ in the following neighborhood of the $n$-th Landau level,

$$
\operatorname{Re} z \in B_{0}[2 n, 2 n+2], \quad|\operatorname{Im} z| \leq B_{0} .
$$


Lemma 3.5 Fix $n \in \mathbb{N}_{0}$ and let $z \in \mathbb{C}$ satisfy (3.16). Suppose (3.3) holds and let $\sigma_{n}:=\left[e_{n, \min }[B], e_{n, \max }[B]\right]$. Then

$$
\left|S_{u, z}(x, y)\right| \leq \frac{\hat{K}_{2} B_{0}^{-1 / 2}+\hat{K}_{3} B_{0}^{-2}}{\operatorname{dist}\left(\operatorname{Re} z, \sigma_{n}\right)} Q_{n}(x, y)
$$

where

$$
Q_{n}(x, y):=\mathcal{Q}_{n}(x-y), \quad \mathcal{Q}_{n}(x):=B_{0} P_{n}\left(B_{0}^{1 / 2}|x|\right) \exp \left(-\frac{1}{8} B_{0}|x|^{2}\right),
$$

and $P_{n}(x)=C_{n}\left(1+|x|^{-1}+|x|^{2(n+1)}\right)$ for some $C_{n}$. The operator $Q_{n}$ is bounded uniformly in $B_{0}$.

Proof. The last sentence is a consequence of (3.17), which can be seen by observing that $\left\|\mathcal{Q}_{n}\right\|_{1}$ and hence the Fourier transform of $\mathcal{Q}_{n}$ are uniformly bounded in $B_{0}$. In the proof we shall write $C$ for a numerical constant which may depend on $n$. For notational simplicity we set

$$
\Pi_{u}=\left(-i \nabla-\widetilde{A}_{u, 0}\right) .
$$

Then

$$
H\left(\widetilde{A}_{u}\right)-H\left(\widetilde{A}_{u, 0}\right)=\left(\delta_{u}[\widetilde{A}]\right)^{2}+i \nabla \cdot \delta_{u}[\widetilde{A}]-2 \delta_{u}[\widetilde{A}] \cdot \Pi_{u}+\delta_{u}[V]
$$

Thus we find

$$
S_{u}=\left(\delta_{u}[\widetilde{A}]\right)^{2} R_{u} \chi_{u, B_{0}}+i\left(\nabla \cdot \delta_{u}[\widetilde{A}]\right) R_{u} \chi_{u, B_{0}}-2 \delta_{u}[\widetilde{A}] \cdot \Pi_{u} R_{u} \chi_{u, B_{0}}+\delta_{u}[V] R_{u} \chi_{u, B_{0}},
$$

where we defined $R_{u}:=\left(z-H_{u}\left(A_{u, 0}\right)\right)^{-1}$ and for simplicity we omitted $z$ from the notation. To estimate the right hand side of (3.18) note first that from (3.8) one has the bounds

$$
\begin{aligned}
& \left|\delta_{u}[\widetilde{A}](x)\right| \leq C\left\|\nabla B_{1}\right\|_{\infty}|x-u|^{2} \\
& \left|\nabla \cdot \delta_{u}[\widetilde{A}](x)\right| \leq C\left\|\nabla B_{1}\right\|_{\infty}|x-u| \\
& \left|\delta_{u}[V]\right| \leq C\|\nabla V\|_{\infty}|x-u| .
\end{aligned}
$$

Moreover, we will use the explicit expression of the integral kernel for the resolvent,

$$
R_{u}(x, y)=\frac{1}{4 \pi}\left[\Gamma_{u} U_{u} E_{u}\right](x, y)
$$

as given in Lemma A.1, where, with $z_{u}:=z-V_{u}$, we define

$$
\begin{aligned}
& \Gamma_{u}(x, y):=\Gamma\left(\frac{1}{2}-\frac{z_{u}}{2 B_{u, 0}}\right), \quad U_{u}(x, y):=U\left(\frac{1}{2}-\frac{z_{u}}{2 B_{u, 0}}, 1 ; \frac{B_{u, 0}}{2}|x-y|^{2}\right), \\
& E_{u}(x, y)=\exp \left(-\frac{B_{u, 0}}{4}|x-y|^{2}-i \frac{B_{u, 0}}{2}[x-u, y-u]\right) .
\end{aligned}
$$

Here $\Gamma(\cdot)$ stands for the usual Gamma-function and $U(\cdot, \cdot ; \cdot)$ is the confluent hypergeometric function. For more details, see Appendix A.

Using this representation, one finds for the two components $\left(\left[\Pi_{u}\right]_{1} R_{u},\left[\Pi_{u}\right]_{2} R_{u}\right)$ of $\Pi_{u} R_{u}$ that

$$
\left[\Pi_{u}\right]_{1,2} R_{u}(x, y)=\left[i \frac{B_{u, 0}}{2}(x-y)_{1,2}+(-1)^{2,1} \frac{B_{u, 0}}{2}(x-y)_{2,1}\right] R_{u}(x, y)-i B_{u, 0}(x-y)_{1,2} R_{u}^{\prime}(x, y),
$$

where we introduced $R_{u}^{\prime}:=\frac{1}{4 \pi} \Gamma_{u} U_{u}^{\prime} E_{u}$ with $U_{u}^{\prime}(x, y)=U_{3}\left(\frac{1}{2}-\frac{z_{u}}{2 B_{u, 0}}, 1 ; \frac{B_{u, 0}}{2}|x-y|^{2}\right)\left(U_{3}\right.$ denotes the partial derivative with respect to the third variable). We conclude that

$$
\left|\left(\Pi_{i} R_{u}\right)(x, y)\right| \leq C B_{u, 0}|x-y|\left[\left|R_{u}(x, y)\right|+\left|R_{u}^{\prime}(x, y)\right|\right], \quad i=1,2 .
$$


Now using (3.22), the bounds (3.19)-(3.21), the triangle inequality $|x-u| \leq|x-y|+|y-u|$, and $\chi_{u, B_{0}}(y) \mid y-$ $u \mid \leq C B_{0}^{-1 / 2}$, to estimate (3.18) we obtain

$$
\begin{aligned}
\left|S_{u}(x, y)\right| \leq & C\left\|\nabla B_{1}\right\|_{\infty}^{2}\left[B_{u, 0}^{-2}\left\{B_{u, 0}|x-y|^{2}\right\}^{2}\left|R_{u}(x, y)\right|+B_{0}^{-2}\left|R_{u}(x, y)\right|\right] \chi_{u, B_{0}}(y) \\
& +C\left\|\nabla B_{1}\right\|_{\infty}\left[B_{u, 0}^{-1 / 2}\left\{B_{u, 0}|x-y|^{2}\right\}^{1 / 2}\left|R_{u}(x, y)\right|+B_{0}^{-1 / 2}\left|R_{u}(x, y)\right|\right. \\
& +B_{u, 0}^{-1 / 2}\left\{B_{u, 0}|x-y|^{2}\right\}^{3 / 2}\left(\left|R_{u}(x, y)\right|+\left|R_{u}^{\prime}(x, y)\right|\right) \\
& \left.+B_{u, 0}^{1 / 2} B_{0}^{-1}\left\{B_{u, 0}|x-y|^{2}\right\}^{1 / 2}\left(\left|R_{u}(x, y)\right|+\left|R_{u}^{\prime}(x, y)\right|\right)\right] \chi_{u, B_{0}}(y) \\
& +C\|\nabla V\|_{\infty}\left[B_{u, 0}^{-1 / 2}\left(B_{u, 0}|x-y|^{2}\right)^{1 / 2}+B_{0}^{-1 / 2}\right]\left|R_{u}(x, y)\right| \chi_{u, B_{0}}(y)
\end{aligned}
$$

Now using (A.3), (A.4), (A.5), and (3.3) to estimate (3.23), we find for $z$ satisfying (3.16),

$$
\left|S_{u}(x, y)\right| \leq \frac{C\left[\hat{K}_{2} B_{0}^{-1 / 2}+\hat{K}_{3} B_{0}^{-2}\right]}{\operatorname{dist}\left(\operatorname{Re} z, \sigma_{n}\right)} B_{u, 0} P_{n}\left(B_{u, 0}^{1 / 2}|x-y|\right) \exp \left(-\frac{1}{4} B_{u, 0}|x-y|^{2}\right) \chi_{u, B_{0}}(y),
$$

with $P_{n}$ of the form as stated in the lemma. Now in view of (3.3) the bound in the lemma now follows.

\section{Location of the Spectrum}

In Subsection 4.2 we prove Theorems 2.2 and 2.3. To this end we first derive in Subsection 4.1 two deterministic results Lemma 4.2 and 4.3

\subsection{Deterministic Part}

The following preparatory Lemma is a trivial consequence of the spectral theorem.

Lemma 4.1 Let $H$ be a self-adjoint operator $E \in \mathbb{R}$ and $\psi \in \mathcal{H}, \psi \neq 0$. Then

$$
\|(H-E) \psi\| \leq \epsilon\|\psi\| \Rightarrow \sigma(H) \cap[E-\epsilon, E+\epsilon] \neq \emptyset .
$$

Proof. Suppose $\sigma(H) \cap[E-\epsilon, E+\epsilon]=\emptyset$. Then by the spectral theorem the assumption implies

$$
\|\psi\|=\left\|(H-E)^{-1}(H-E) \psi\right\|<\frac{1}{\epsilon} \epsilon\|\psi\|,
$$

which is a contradiction.

The following Lemma holds for an arbitrary magnetic field $B$, which is bounded from below. It will be used in the proof of Theorem 2.2.

Lemma 4.2 Let $B(x)$ be a magnetic field with

$$
B_{\text {inf }}:=\inf _{x} B(x)>0 .
$$

Then for any $n \in \mathbb{N}_{0}$ there exists a constant $C_{n}$ (depending on $n$ ) such that for all $\lambda$ in the range of the function $(2 n+1) B+V$,

$$
\operatorname{dist}(\sigma(H(B)), \lambda) \leq C_{n}\left(\left[\|\nabla B\|_{\infty}+\|\nabla V\|_{\infty}\right] B_{\mathrm{inf}}^{-1 / 2}+\|\nabla B\|_{\infty}^{2} B_{\mathrm{inf}}^{-2}\right) .
$$


Proof. By assumption, there exists an $\widetilde{x} \in \mathbb{R}^{2}$ such that $\lambda=V(\widetilde{x})+(2 n+1) B(\widetilde{x})$. Choose a gauge

$$
A(x)=\frac{1}{2}\left(-\int_{\widetilde{x}_{2}}^{x_{2}} B\left(x_{1}, y_{2}\right) \mathrm{d} y_{2}, \int_{\widetilde{x}_{1}}^{x_{1}} B\left(y_{1}, x_{2}\right) \mathrm{d} y_{1}\right),
$$

and set $A_{0}(x):=\frac{1}{2} B_{0}\left(-\left(x_{2}-\widetilde{x}_{2}\right), x_{1}-\widetilde{x}_{1}\right)$ with $\widetilde{B}_{0}:=B(\widetilde{x})$. Let us consider the normalized trial state

$$
\varphi_{n}(x)=\left\{\frac{\widetilde{B}_{0}}{2 \pi}\right\}^{1 / 2} L_{n}\left(\widetilde{B}_{0}|x-\widetilde{x}|^{2} / 2\right) \exp \left(-\frac{1}{4} \widetilde{B}_{0}|x-\widetilde{x}|^{2}\right),
$$

where $L_{n}$ is the $n$-th Laguerre polynomial. We set

$$
\delta[A]:=A-A_{0}, \quad \delta[V]:=V-V(\widetilde{x}) .
$$

Expanding the square, one finds

$$
\begin{aligned}
H(A) \varphi_{n} & =H\left(A_{0}\right) \varphi_{n}+\left[H(A)-H\left(A_{0}\right)\right] \varphi_{n} \\
& =\lambda \varphi_{n}+\left\{\delta[V]+(\delta[A])^{2}+i \nabla \cdot \delta[A]-2 \delta[A] \cdot \widetilde{\Pi}\right\} \varphi_{n},
\end{aligned}
$$

with $\widetilde{\Pi}:=\left(p-A_{0}\right)$. Using the estimates

$$
\begin{aligned}
|\delta[A](x)| & \leq C\|\nabla B\|_{\infty}|x-\tilde{x}|^{2} \\
|\nabla \cdot \delta[A](x)| & \leq C\|\nabla B\|_{\infty}|x-\tilde{x}| \\
|\delta[V]| & \leq\|\nabla V\|_{\infty}|x-\widetilde{x}|,
\end{aligned}
$$

and (4.2), we find that

$$
\left\|H(A) \varphi_{n}-\lambda \varphi_{n}\right\| \leq C\left[\frac{\|\nabla B\|_{\infty}+\|\nabla V\|_{\infty}}{\widetilde{B}_{0}^{1 / 2}}+\frac{\|\nabla B\|_{\infty}^{2}}{\widetilde{B}_{0}^{2}}\right] .
$$

The lemma now follows in view of Lemma 4.1 and since by definition $\widetilde{B}_{0} \geq B_{\text {inf }}$.

The following Lemma estimates the change of the spectrum of a magnetic Schrödinger with arbitrary magnetic field $B$ under a the perturbation by a small magnetic field $B^{\prime}$. It will be used in the proof of Theorem 2.3.

Lemma 4.3 Let $\epsilon>0, K>1$, and $v>0$. Then there exists an $\eta>0$ such that the following holds. For any magnetic field $B$, potential $V$, with $\|V\|_{\infty} \leq v$, energy $E \in \sigma(H(B))$, with $E+1 \leq K$, and magnetic field $B^{\prime}$, with $\left\|B^{\prime}\right\|+\left\|\nabla B^{\prime}\right\| \leq \eta$, one has

$$
[E-\epsilon, E+\epsilon] \cap \sigma\left(H\left(B+B^{\prime}\right)\right) \neq \emptyset .
$$

The crucial part of the lemma is that the $\eta$ does not depend on the magnetic field $B$.

Proof. First, we will show that there exists a trial state which is localized in a box of finite side length $L$ (depending only on $\epsilon, K$, and $v$ ), see (4.11). Then we can use perturbation theory to complete the proof.

We consider the following partition of $\mathbb{R}^{2}$. Let $\chi$ be a smooth function with support contained in $B_{2}(0)=\left\{x \in \mathbb{R}^{2}:|x| \leq 2\right\}$ such that $\sum_{z \in \mathbb{Z}^{2}} \chi_{z}^{2}(x)=1$, where $\chi_{z}(x)=\chi(x-z)$. We assume that at most four different $\chi_{z}$ 's overlap, that is

$$
\sum_{z} 1_{\operatorname{supp} \chi_{z}} \leq 4
$$


Set $\chi_{z, L}(x)=\chi\left(L^{-1}(x-z)\right)$. Without loss we can assume that $0<\epsilon \leq 1$. Let $\psi$ be a normalized state such that

$$
\|(H(A)-E) \psi\| \leq \frac{\epsilon}{100} .
$$

Choose $L$ sufficiently large such that

$$
\frac{80\|\nabla \chi\|_{\infty}^{2}}{L^{2}}(K+v) \leq \frac{\epsilon^{2}}{100}, \quad \frac{40\|\Delta \chi\|_{\infty}^{2}}{L^{4}} \leq \frac{\epsilon^{2}}{100} .
$$

We claim, that there exists a $z \in \mathbb{Z}^{2}$ such that

$$
\begin{aligned}
\left\|\chi_{L, z} \psi\right\|^{2} & \geq \frac{L^{2}}{80\|\nabla \chi\|_{\infty}^{2}(K+v)}\left\|\nabla \chi_{L, z} \cdot \Pi \psi\right\|^{2} \\
\left\|\chi_{L, z} \psi\right\|^{2} & \geq \frac{L^{4}}{40\|\Delta \chi\|_{\infty}^{2}}\left\|\Delta \chi_{L, z} \psi\right\|^{2} \\
\left\|\chi_{L, z} \psi\right\|^{2} & \geq \frac{100}{\epsilon^{2}}\left\|\chi_{L, z} \xi\right\|^{2}
\end{aligned}
$$

where $\xi:=(H-E) \psi$ and $\Pi:=p-A$. Suppose this were not the case: then for all $z \in \mathbb{Z}^{2}$ one of the inequalities (4.7)-(4.9) would not hold and this would imply the first inequality of the following estimate

$$
\begin{aligned}
1 & =\sum_{z \in \mathbb{Z}^{2}}\left\|\chi_{L, z} \psi\right\|^{2} \\
& \leq \frac{L^{2}}{80\|\nabla \chi\|_{\infty}^{2}(K+v)} \sum_{z}\left\|\nabla \chi_{L, z} \cdot \Pi \psi\right\|^{2}+\frac{L^{4}}{40\|\Delta \chi\|_{\infty}^{2}} \sum_{z}\left\|\Delta \chi_{L, z} \psi\right\|^{2}+\frac{100}{\epsilon^{2}} \sum_{z}\left\|\chi_{L, z} \xi\right\|^{2} \\
& \leq \frac{4}{10},
\end{aligned}
$$

where the first term on the second line were estimated by $1 / 10$ as follows,

$$
\begin{aligned}
\sum_{z}\left\|\nabla \chi_{L, z} \cdot \Pi \psi\right\|^{2} & \leq \sum_{z} 2 \sum_{i=1}^{2}\left(\Pi_{i} \psi,\left(\nabla_{i} \chi_{L, z}\right)^{2} \Pi_{i} \psi\right) \\
& \leq 8\|\nabla \chi\|_{\infty}^{2} L^{-2} \sum_{i=1}^{2}\left(\Pi_{i} \psi, \Pi_{i} \psi\right)=8\|\nabla \chi\|_{\infty}^{2} L^{-2}[(\psi, H(A) \psi)+v] \\
& \leq 8\|\nabla \chi\|_{\infty}^{2} L^{-2}(K+v),
\end{aligned}
$$

where we used (4.4) in the second line and (4.5) in the last. The other terms in the second line of (4.10) can be estimated in a similar but easier way. But (4.10) yields a contradiction. Thus let $z^{*} \in \mathbb{Z}^{2}$ be such that (4.7)-(4.9) hold. Then calculating a commutator, using the triangle inequality, we find

$$
\begin{aligned}
\left\|(H(A)-E) \chi_{L, z^{*}} \psi\right\| & =\left\|\chi_{L, z^{*}} \xi-2 i \nabla \chi_{L, z^{*}} \cdot \Pi \psi-\Delta \chi_{L, z^{*}} \psi\right\| \\
& \leq\left\|\chi_{L, z^{*}} \xi\right\|+2\left\|\nabla \chi_{L, z^{*}} \cdot \Pi \psi\right\|+\left\|\Delta \chi_{L, z^{*}} \psi\right\| \leq \frac{\epsilon}{2}\left\|\chi_{L, z^{*}} \psi\right\|,
\end{aligned}
$$

where the last inequality is a consequence of (4.7)-(4.9) and the choice of $L,(4.6)$.

Now we can use ordinary perturbation theory. We choose the gauge

$$
A^{\prime}\left(x_{1}, x_{2}\right):=\left(-\frac{1}{2} \int_{z_{2}^{*}}^{x_{2}} B^{\prime}\left(x_{1}, y\right) d y, \frac{1}{2} \int_{z_{1}^{*}}^{x_{1}} B^{\prime}\left(y, x_{2}\right) d y\right) .
$$

Let $\varphi=\chi_{L, z^{*}} \psi$ and let $G$ denote the support of $\chi_{L, z^{*}}$. Then by (4.12) we have

$$
\left\|\left(A^{\prime}\right)_{j} \mathbf{1}_{G}\right\|_{\infty} \leq\left\|B^{\prime}\right\|_{\infty} L, \quad\left\|\left(A^{\prime}\right)^{2} \mathbf{1}_{G}\right\|_{\infty} \leq 2\left\|B^{\prime}\right\|_{\infty}^{2} L^{2}, \quad\left\|\nabla \cdot A^{\prime} \mathbf{1}_{G}\right\|_{\infty} \leq\left(\left\|B^{\prime}\right\|_{\infty}+2\left\|\nabla B^{\prime}\right\|_{\infty} L\right) .
$$


We find, using first the triangle inequality and then (4.11) and (4.13),

$$
\begin{aligned}
& \left\|\left[\left(p-A-A^{\prime}\right)^{2}+V-E\right] \varphi\right\| \\
& \quad \leq\left\|\left[(p-A)^{2}+V-E\right] \varphi\right\|+\left\|\left[A^{\prime 2}+i \nabla \cdot A^{\prime}\right] \varphi\right\|+\left\|2 A^{\prime} \cdot(p-A) \varphi\right\| \\
& \quad \leq\left[\frac{\epsilon}{2}+2\left\|B^{\prime}\right\|_{\infty}^{2} L^{2}+\left\|B^{\prime}\right\|_{\infty}+2\left\|\nabla B^{\prime}\right\|_{\infty} L\right]\|\varphi\|+2\left\|B^{\prime}\right\|_{\infty} L\left[\left\|(p-A)_{1} \varphi\right\|+\left\|(p-A)_{2} \varphi\right\|\right] .
\end{aligned}
$$

Now inserting the estimate

$$
\left[\left\|(p-A)_{1} \varphi\right\|+\left\|(p-A)_{2} \varphi\right\|\right]^{2} \leq 2\left(\varphi,(p-A)^{2} \varphi\right) \leq 2(K+v)\|\varphi\|^{2},
$$

into (4.14) we find

$$
\left\|\left[\left(p-A-A^{\prime}\right)^{2}+V-E\right] \varphi\right\| \leq \epsilon\|\varphi\|,
$$

provided we choose $\eta>0$ sufficiently small (depending only on $K$ and $L$ ). The Lemma now follows in view of Lemma 4.1.

\subsection{Probabilistic Part}

To show Theorems 2.2 and 2.3, we will combine the previous two Lemmas concerning a deterministic magnetic field, with the following probabilistic result [5, Theorem 8.1].

Theorem 4.4 Suppose $B_{\omega}$ is a random magnetic field constructed in (2.1), (2.2), and (2.4), satisfying (2.3) and (i.i.d.) and $\rho>\ln 2$. Assume $B_{\mathrm{var}}$ and $V$ are $\mathbb{Z}^{2}$-periodic. Then for all $\omega$ in the support of the probability measure we have

$$
\Sigma \supset \sigma\left(H\left(B_{\omega}\right)\right) .
$$

Proof of Theorem 2.2. The theorem follows since by Theorem 4.4 we have $\sigma\left(H\left(A_{\omega^{ \pm}}\right)\right) \subset \Sigma$ and Lemma 4.2 .

Proof of Theorem 2.3. By Theorem 2.1 there exists an $\omega^{*}$ such that $\Sigma=\sigma\left(H\left(A_{\omega^{*}}\right)\right)$. In particular, it follows that $\Sigma$ is a closed set. Let $E \in \Sigma$ and let $\epsilon>0$. Now we consider the path $\omega_{t}=t \omega^{*}$, with $t \in[0,1]$. Using Lemma 4.3, we can find a sufficiently large $N$ such that for the numbers $t_{i}=1-\frac{i}{N}$, with $i=1, \ldots, N$, there exist $E_{i} \in \sigma\left(H_{\omega_{t_{i}}}\right)$ satisfying $\left|E_{i}-E_{i-1}\right| \leq \epsilon$. Clearly, $E_{N} \in\left\{B_{0}(2 n+1): n \in \mathbb{N}\right\}$. By Theorem 4.4, we know that $E_{i} \in \Sigma$. Since $\epsilon>0$ can be chosen arbitrarily small by choosing $N$ sufficiently large, it follows that all numbers between $E$ and $B_{0}(2 n+1)$, for some $n \in \mathbb{N}_{0}$, are contained in $\Sigma$, since $\Sigma$ is closed.

This implies the existence of the intervals as stated in the theorem. It remains to show the estimate regarding the endpoints of $\Sigma_{n}$. We will set

$$
C_{n}=\max \left(C_{\mathrm{int}, n}, C_{\mathrm{ext}, n}\right) .
$$

Fix $n$. By Theorem 3.2 we can choose $B_{0}$ sufficiently large such that there is a gap in the spectrum $\Sigma$ located between the Landau levels $B_{0}(2 n+1)$ and $B_{0}(2 n+3)$ and that $E_{\min , n}$ is to the left of the gap and $E_{\max , n+1}$ is to the right of the gap. The estimate (2.10) regarding the endpoints of $\Sigma_{n}$ can be rephrased as two inequalities. One of the inequalities follows in view of (3.2) from Theorem 2.2 and the other from Theorem 3.2 . 


\section{Localization}

In this section we show Theorem 2.5 using multi-scale analysis and the Wegner estimate from [5]. We have to show the initial length scale estimate, which is the content of Subsections 5.1, 5.2, and 5.3.

By $\Lambda \subset \mathbb{R}^{2}$ we denote a square. We will consider the magnetic Schrödinger operator with Dirichlet boundary conditions on $\Lambda$, and denote it by

$$
H_{\Lambda}(A)=(p-A)^{2}+V .
$$

We realize this as a self adjoint operator by means of the Friedrichs extension. We will work in the Hilbert space $L^{2}(\Lambda)$ and denote the scalar product by $\langle\cdot, \cdot\rangle$ and the norm by $\|\cdot\|$. In particular we will work with the following squares. For $l>0$ and $x \in \mathbb{R}^{2}$ we denote by

$$
\Lambda_{l}(x):=\left\{y \in \mathbb{R}^{2}:|y-x|_{\infty}<l / 2\right\}
$$

the open square centered at $x$ with sidelength $l$.

If $l \in \mathbb{N}$, we will write

$$
H_{l}(A)=H_{\Lambda_{l}(0)}(A) .
$$

Boxes with sidelength $l \in 2 \mathbb{N}+1$ and center $x \in \mathbb{Z}^{2}$ are called suitable. For suitable squares, we set

$$
\Lambda^{\text {int }}:=\Lambda_{l / 3}(x), \quad \Lambda^{\text {out }}:=\Lambda_{l}(x) \backslash \Lambda_{l-2}(x),
$$

and we set $\chi^{\text {int }}=\chi_{\Lambda^{\text {int }}}$ and $\chi^{\text {out }}=\chi_{\Lambda^{\text {out }}}$.

We introduce the constant $c_{\delta}$ to be the smallest integer such that

$$
c_{\delta} \geq \begin{cases}\frac{3}{2}, & \text { in case }(2.11) \\ \delta^{-1}, & \text { in case }(2.12)\end{cases}
$$

which gives the distance beyond which the random magnetic field is independent. We define $\widetilde{\Lambda}:=\Lambda+$ $\left[-c_{\delta}, c_{\delta}\right]^{2}$.

In this section we consider the random magnetic field $B_{\omega}=B_{\text {det }}+\mu B_{\text {ran }}^{\omega}$, as introduced in Section 2. Let $A_{\omega}$ be a vector potential with $\nabla \times A_{\omega}=B_{\text {det }}+\mu B_{\text {ran }}^{\omega}$. We introduce a random magnetic field subordinate to the square $\Lambda$,

$$
\widetilde{B}_{\Lambda, \omega}:=B_{\mathrm{det}}+\mu \widetilde{B}_{\mathrm{ran}, \Lambda}^{\omega}, \quad \widetilde{B}_{\mathrm{ran}, \Lambda}^{\omega}:=\sum_{k=0}^{\infty} \sum_{z \in \Lambda^{(k)} \cap \widetilde{\Lambda}} B_{z}^{(k)} ;
$$

for notation we refer the reader to (2.4). Informally speaking, the random field (5.5) is obtained by adding to the deterministic magnetic field the random magnetic field generated only by the random variables living on the square $\widetilde{\Lambda}$. Likewise, for a random vector potential $A_{\omega}$ generating the magnetic field $B_{\omega}$, we introduce a random vector potential subordinated to the square $\Lambda$, centered at $z$, by

$$
\begin{aligned}
& \widetilde{A}_{\Lambda, \omega}(x):=A_{\omega}(x)-\left(-\frac{1}{2} \int_{z_{2}}^{x_{2}} \delta\left[\widetilde{B}_{\Lambda, \omega}\right]\left(x_{1}, \xi\right) d \xi, \frac{1}{2} \int_{z_{1}}^{x_{1}} \delta\left[\widetilde{B}_{\Lambda, \omega}\right]\left(\xi, x_{2}\right) d \xi\right) \\
& \delta\left[\widetilde{B}_{\Lambda, \omega}\right]:=B_{\omega}-\widetilde{B}_{\Lambda, \omega} .
\end{aligned}
$$

Observe that $\widetilde{A}_{\Lambda, \omega}$ is a vector potential with magnetic field $\widetilde{B}_{\Lambda, \omega}$ such that $\widetilde{A}_{\Lambda, \omega}=A_{\omega}$ on $\Lambda$.

For an operator $T$ in a Hilbert space we will denote by $\rho(T)$ the resolvent set of $T$.

\subsection{The Wegner Estimate and Exponential Decay}

In this subsection we first state the Wegner estimate from [5]. Fix an energy $E$ and a window of width $\eta \leq 1$ about $E$. Let $\chi_{E, \eta}$ be the characteristic function of the interval $[E-\eta / 2, E+\eta / 2]$. 
Theorem 5.1 [5, Theorem 3.1] Let (W) hold with $K_{0}>3, \rho>\ln 2$ and $0<\delta \leq \delta_{0}$. Let $K_{1} \geq 1$. Then there exist positive constants $C_{0}=C_{0}\left(K_{0}, K_{1}\right), C_{1}=C_{1}\left(K_{0}, K_{1}\right)$, and $L_{0}^{*}=L_{0}^{*}\left(K_{0}, K_{1}, \delta\right)$ such that for any $0<\kappa \leq 1$

$$
\mathbb{E} \operatorname{Tr} \chi_{E, \eta}\left(H_{l}(A)\right) \leq C_{0} \eta \mu^{-2} l^{C_{1}\left(\kappa^{-1}+\rho\right)},
$$

for all $E \in\left[\frac{b_{0}}{2}, K_{1} b_{0}\right], 0<\eta \leq 1$, and $l \geq L_{0}^{*} b_{0}^{\kappa}$.

Now we use the Wegner estimate and the geometric resolvent identity to show the following Lemma. It will be used to show that for two given independent squares (squares which are sufficiently far apart such that their Hamiltonians are independent) with very high probability for at least one of the squares the exponential decay of the finite volume Hamiltonian can be estimated in terms of the infinite volume Hamiltonian. In precise terms, we say a square $\Lambda=\Lambda_{l}(x)$ is $\left(E, C_{\infty}, \alpha\right)$-balanced if $E \in \rho\left(\left(H_{\Lambda}(A)\right)\right.$ and

$$
\left\|\chi^{\text {out }}\left(H_{\Lambda}(A)-E\right)^{-1} \chi^{\text {int }}\right\| \leq\left(1+C_{\infty} l^{\alpha}\right)\left\|\chi^{\text {out }}\left(H\left(\widetilde{A}_{\Lambda}\right)-E\right)^{-1} \chi^{\text {int }}\right\| .
$$

Lemma 5.2 Let (W) hold with $K_{0}>3$, and let $K_{1} \geq 1$ and $0<\kappa \leq 1$. There exist constants $C_{0}, C_{1}, L_{0}^{*}$ (the same as in Theorem 5.1) and a constant $C_{\infty}=C_{\infty}\left(K_{1}\right)$, such that for any $\alpha>0$, subinterval $J \subset\left[\frac{b_{0}}{2}, K_{1} b_{0}\right]$ and any $x, y \in \mathbb{Z}^{2}$ with $|x-y|_{\infty} \geq l+c_{\delta}$,

$$
\mathbb{P}\left(\forall E \in J, \Lambda_{l}(x) \text { or } \Lambda_{l}(y) \text { is }\left(E, b_{0} C_{\infty}, \alpha\right) \text {-balanced }\right) \geq 1-3 C_{0}^{2}|J| \mu^{-4} l^{2 C_{1}\left(\kappa^{-1}+\rho\right)} l^{-\alpha},
$$

provided $l \geq L_{0}^{*} b_{0}^{\kappa}$.

Proof. To shorten notation we set $\beta=C_{1}\left(\kappa^{-1}+\rho\right)$ and $D_{0}=C_{0} \mu^{-2}$.

Step 1: Let $E \in J$. Then we claim that with probability greater or equal than the right hand side of (5.7) we have for $w=x$ or $w=y$ that $E \in \rho\left(H_{\Lambda(w)}\right)$ and

$$
\left\|\left(H_{\Lambda(w)}(A)-E\right)^{-1}\right\| \leq 8 l^{\alpha} .
$$

To prove (5.8), let $a$ denote the left endpoint of the interval $J$ and we consider a partition of $J$ with respect to the points

$$
x_{j}=a+j l^{-\alpha} .
$$

Define the intervals $J_{j, 1}=\left[x_{2 j-1}, x_{2 j+1}\right]$, and $J_{j, 2}=\left[x_{2 j}, x_{2 j+2}\right]$. Then

$$
J \subset \bigcup_{j=0}^{N}\left(J_{j, 1} \cup J_{j, 2}\right),
$$

where $N$ denotes the smallest integer larger than $|J| l^{\alpha} / 2$. Using the independence of spectral properties of the local Hamiltonians in $\Lambda(x)$ and $\Lambda(y)$ in addition to the Wegner estimate, we find

$$
\mathbb{P}\left\{\forall_{w \in\{x, y\}} J_{j, \sigma} \cap \sigma\left(H_{\Lambda(w)}\right) \neq \emptyset\right\}=\prod_{w \in\{x, y\}} \mathbb{P}\left\{J_{j, \sigma} \cap \sigma\left(H_{\Lambda(w)}\right) \neq \emptyset\right\} \leq D_{0}^{2} l^{-2 \alpha+2 \beta} .
$$

Since the covering of $J$ given in (5.9) contains $2 N\left(\leq 3|J| l^{\alpha}\right)$ intervals, we have

$$
\mathbb{P}\left\{\exists_{j \in\{1,2, . ., N\}} \exists_{\sigma \in\{1,2\}} \forall_{w \in\{x, y\}} J_{j, \sigma} \cap \sigma\left(H_{\Lambda(w)}\right) \neq \emptyset\right\} \leq 3|J| D_{0}^{2} l^{-\alpha+2 \beta} .
$$

Thus the probability that this event does not occur can be estimated from below,

$$
\mathbb{P}\left\{\forall_{j \in\{1,2, . ., N\}} \forall_{\sigma \in\{1,2\}} \exists_{w \in\{x, y\}} J_{j, \sigma} \cap \sigma\left(H_{\Lambda(w)}\right)=\emptyset\right\} \geq 1-3|J| D_{0}^{2} l^{-\alpha+2 \beta}
$$


By (5.9) for any $E \in J$ there exists an interval $J_{j, \sigma}$ which contains $E$ such that the distance of $E$ to the boundary of $J_{j, \sigma}$ is greater than $l^{-\alpha} / 8$. This observation and (5.10) imply the claim in Step 1.

Step 2: Step 1 implies (5.7).

For $w=x$ or $w=y$ we write $\Lambda=\Lambda(w)$. By the geometric resolvent identity, we have

$$
\chi^{\text {out }}\left(H_{\Lambda}(A)-E\right)^{-1} \chi^{\text {int }}=\chi^{\text {out }} \phi\left(H\left(\widetilde{A}_{\Lambda}\right)-E\right)^{-1} \chi^{\text {int }}+\chi^{\text {out }}\left(H_{\Lambda}(A)-E\right)^{-1} W(\phi)\left(H\left(\widetilde{A}_{\Lambda}\right)-E\right)^{-1} \chi^{\text {int }},
$$

with $W(\phi)=2 \nabla \phi \cdot\left(p-\widetilde{A}_{\Lambda}\right)+\Delta \phi$. We choose $\phi \in C_{0}^{\infty}\left(\mathbb{R}^{2} ;[0,1]\right)$ to be a function such that

$$
\phi=1 \quad \text { on } \quad \Lambda_{l-1}, \quad \phi=0 \quad \text { on } \quad \mathbb{R}^{2} \backslash \Lambda_{l-1 / 2} .
$$

To estimate the second term in (5.11) we use

$$
\left\|(\Delta \phi)\left(H\left(\widetilde{A}_{\Lambda}\right)-E\right)^{-1} \chi^{\mathrm{int}}\right\| \leq C\left\|\chi^{\mathrm{out}}\left(H\left(\widetilde{A}_{\Lambda}\right)-E\right)^{-1} \chi^{\mathrm{int}}\right\|,
$$

and we use Lemma B.1 of [5] with $u=\left(H\left(\widetilde{A}_{\Lambda}\right)-E\right)^{-1} \chi^{\text {int }}, \widetilde{\Omega}=\operatorname{supp}|\nabla \phi|, \Omega=\Lambda^{\text {out }}$, yielding

$$
\left\|\nabla \phi \cdot\left(p-\widetilde{A}_{\Lambda}\right)\left(H\left(\widetilde{A}_{\Lambda}\right)-E\right)^{-1} \chi^{\mathrm{int}}\right\| \leq C(1+|E|)\left\|\chi^{\mathrm{out}}\left(H\left(\widetilde{A}_{\Lambda}\right)-E\right)^{-1} \chi^{\mathrm{int}}\right\|
$$

using that $\chi^{\text {int }}=0$ on $\Omega$. Now using (5.12), (5.13) and (5.8) to estimate (5.11) Step 2 and hence the lemma follows, since $E \in\left[\frac{b_{0}}{2}, K_{1} b_{0}\right]$.

\subsection{Lifshitz asymptotics}

In this subsection we show a Lifshitz estimate for the probability that the operator $H\left(\widetilde{A}_{\Lambda}\right)$ with a local magnetic field has an eigenvalue beyond the outer bound on the infinite volume operator. This result will imply a spectral estimate in Corollary 5.4 which will be used in the next section to obtain the initial length scale estimate. In the theorem below we will use similar notation as introduced in Theorem (3.2), but we take the supremum over the essential support of the probability measure. That is we define $I_{n}[B]:=$ $\left(I_{n}^{+}[B], I_{n+1}^{-}[B]\right)$ with

$$
\begin{aligned}
I_{n}^{+}[B] & :=e_{n, \max }[B]+C_{\text {ext }, n}\left[K_{2} B_{0}^{-1 / 2}+K_{3} B_{0}^{-2}\right], \\
I_{n+1}^{-}[B] & :=e_{n+1, \min }[B]-C_{\text {ext }, n+1}\left[K_{2} B_{0}^{-1 / 2}+K_{3} B_{0}^{-2}\right],
\end{aligned}
$$

with $K_{3}:=\operatorname{ess}_{\sup }\left\|B_{\omega}\right\|_{\infty}^{2}$.

Theorem 5.3 Assume that $\left(\mathbf{A}_{\boldsymbol{\tau}}\right)$ holds. Then for $h>0$ the probability that

$$
\left(I_{n}^{+}\left[B_{\omega_{+}}\right]-(2 n+1) \mu h, I_{n+1}^{-}\left[B_{\omega_{-}}\right]+(2 n+3) \mu h\right) \subset \rho\left(H\left(\widetilde{A}_{\Lambda}\right)\right)
$$

holds, satisfies the lower bound

$$
\mathbb{P}\{(5.16)\} \geq 1-|\widetilde{\Lambda}|\left[\nu_{+}\left(c_{u}^{-1} h\right)+\nu_{-}\left(c_{u}^{-1} h\right)\right] .
$$

For the sake of a transparent exposition, we set $\left(m_{+}\right)_{z}^{(k)}:=\left(\omega_{+}\right)_{z}^{(k)}$.

Proof. Consider the events

$$
\begin{aligned}
& \omega_{z}^{0} \leq m_{+}^{(0)}-c_{u}^{-1} h, \quad z \in \widetilde{\Lambda} \\
& \omega_{z}^{0} \geq m_{-}^{(0)}+c_{u}^{-1} h, \quad z \in \widetilde{\Lambda}
\end{aligned}
$$


If event (5.18) holds, then

$$
\begin{aligned}
(2 n+1) \widetilde{B}_{\Lambda}(x)+V(x) & =(2 n+1)\left[B_{\operatorname{det}}(x)+\mu \sum_{k=0}^{\infty} \sum_{z \in \widetilde{\Lambda}^{(k)}} \omega_{z}^{(k)} u(x-z)\right]+V(x) \\
& \leq(2 n+1)\left[B_{\operatorname{det}}(x)-\mu \sum_{z \in \widetilde{\Lambda}^{(0)}} c_{u}^{-1} h u(x-z)+\mu \sum_{k=0}^{\infty} \sum_{z \in \widetilde{\Lambda}^{(k)}} m_{+}^{(k)} u(x-z)\right]+V(x) \\
& \leq(2 n+1)\left[B_{\operatorname{det}}(x)-\mu h+\mu \sum_{k=0}^{\infty} \sum_{z \in \widetilde{\Lambda}^{(k)}} m_{+}^{(k)} u(x-z)\right]+V(x) \\
& \leq e_{n, \max }\left(B_{\omega_{+}}\right)-(2 n+1) \mu h,
\end{aligned}
$$

where we used (2.15) from the second to third line. By this and the definition in (3.4) it follows that the event (5.18) implies

$$
I_{n}^{+}\left[\widetilde{B}_{\Lambda}\right] \leq I_{n}^{+}\left[B_{\omega_{+}}\right]-(2 n+1) \mu h .
$$

Similarly one can show that the event (5.19) implies

$$
I_{n+1}^{-}\left[\widetilde{B}_{\Lambda}\right] \geq I_{n+1}^{-}\left[B_{\omega_{-}}\right]+(2 n+3) \mu h .
$$

On the other hand, by Theorem 3.2 it follows that (5.20) and (5.21) imply (5.16). Thus it remains to estimate the following probability,

$$
\begin{aligned}
\mathbb{P}\{(5.18) \text { and }(5.19)\} & =\left[\mathbb{P}\left\{\omega_{0}^{(0)} \leq m_{+}^{(0)}-c_{u}^{-1} h \text { and } \omega_{0}^{(0)} \geq m_{-}^{(0)}+c_{u}^{-1} h\right\}\right]^{|\widetilde{\Lambda}|} \\
& \geq 1-|\widetilde{\Lambda}|\left(\nu_{+}\left(c_{u}^{-1} h\right)+\nu_{-}\left(c_{u}^{-1} h\right)\right),
\end{aligned}
$$

where the last line follows from the binomial formula.

Corollary 5.4 Assume that $\left(\mathbf{A}_{\tau}\right)$ holds for some fixed $\tau>2$ and $c_{v}$. For any $\xi \in(0, \tau-2)$ set $\beta:=$ $\frac{1}{2}\left(1-\frac{\xi+2}{\tau}\right) \in(0,1)$, then there is an $l_{\text {initial }}=l_{\text {initial }}\left(\tau, \xi, c_{u}, c_{v}, c_{\delta}\right)$ such that

$$
\mathbb{P}\left\{\operatorname{dist}\left(\sigma\left(H\left(\widetilde{A}_{\Lambda}\right),\left(I_{n}^{+}\left[B_{\omega_{+}}\right], I_{n+1}^{-}\left[B_{\omega_{-}}\right]\right)\right) \geq(2 n+1) \mu l^{\beta-1}\right\} \geq 1-\frac{1}{4} l^{-\xi}\right.
$$

for any $\Lambda=\Lambda_{l}(x)$, with $x \in \mathbb{Z}^{2}$ and $l \geq l_{\text {initial }}$ (we adopt the convention that the distance to the empty set is infinity).

Proof. Set $h=l^{\beta-1}$ in Theorem 5.3. Then

$$
|\widetilde{\Lambda}|\left[\nu_{+}\left(c_{u}^{-1} h\right)+\nu_{-}\left(c_{u}^{-1} h\right)\right] \leq|\widetilde{\Lambda}| 2 c_{v}\left(c_{u}^{-1} h\right)^{\tau}=2 c_{u}^{-\tau} c_{v}\left(l+c_{\delta}\right)^{2} l^{(\beta-1) \tau} \leq \frac{1}{4} l^{-\xi},
$$

where the first inequality follows from assumption $\left(\mathbf{A}_{\tau}\right)$, and the second inequality holds for large $l$.

\subsection{Multi-scale Analysis: Proof of Theorem 2.5}

For the proof of Theorem 2.5, we will essentially follow the argument in [5] that is based on [14] after including the magnetic field.

We assume $\left(\mathbf{A}_{\boldsymbol{\tau}}\right)$ throughout this section for some fixed $\tau>2$ and $c_{v}$. The constants $b_{0}, \rho, \delta$ are as in the assumptions of Theorem 5.1. 
Definition 5.5 A square $\Lambda$ is called $(\gamma, E)$-good for $\omega \in \Omega$ if

$$
\left\|\chi^{\text {out }}\left(H_{\Lambda}\left(A_{\omega}\right)-E\right)^{-1} \chi^{\text {int }}\right\| \leq \exp (-\gamma l),
$$

where $E \in \rho\left(H_{\Lambda}\left(A_{\omega}\right)\right)$.

Let us introduce the multiscale induction hypotheses. Below we denote by $I \subset \mathbb{R}$ an interval and assume $l \in 2 \mathbb{N}+1$. First, for $\gamma>0$, and $\xi>0$ we introduce the following hypothesis.

$G(I, l, \gamma, \xi): \quad \forall x, y \in \mathbb{Z}^{2},|x-y|_{\infty} \geq l+c_{\delta}$, the following estimate holds:

$$
\mathbb{P}\left\{\forall E \in I, \Lambda_{l}(x) \text { or } \Lambda_{l}(y) \text { is }(\gamma, E)-\operatorname{good}\right\} \geq 1-l^{-2 \xi} .
$$

Note that this definition includes a security distance $c_{\delta}$, to ensure the independence of squares.

Lemma 5.6 Fix $n \in \mathbb{N}$. For any $\xi \in\left(0, \frac{\tau}{2}-1\right)$ there is an $l_{G}=l_{G}\left(\tau, \xi, c_{u}, c_{v}, c_{\delta}, K_{0}, K_{1}, \delta, \mu, \kappa, \rho, n\right)$ such that for all $l \geq l_{G} b_{0}^{\kappa}, G(I, l, \gamma, \xi)$ holds with $\gamma=l^{\beta-1}, \beta=\frac{1}{2}\left(1-\frac{2 \xi+2}{\tau}\right) \in(0,1)$, and if $I$ is any of the intervals of the form

$$
\begin{aligned}
& I=I_{n}^{+}\left[B_{\omega_{+}}\right]+\left[-\frac{1}{2} \mu l^{\beta-1}, 0\right] \\
& I=I_{n}^{-}\left[B_{\omega_{-}}\right]+\left[0, \frac{1}{2} \mu l^{\beta-1}\right]
\end{aligned}
$$

as long as $I \subset\left[\frac{b_{0}}{2}, K_{1} b_{0}\right]$.

Proof. First we give a deterministic estimate and then we estimate the probability.

Let $A$ be a vector potential and let $\Lambda=\Lambda_{l}$. Suppose the vector potential satisfies

$$
\operatorname{dist}\left(\sigma\left(H\left(\widetilde{A}_{\Lambda}\right)\right), I_{n}^{ \pm}\left[B_{\omega_{ \pm}}\right]\right) \geq(2 n+1) \mu l^{\beta-1} .
$$

If $E \in I$, then $\operatorname{dist}\left(H\left(\widetilde{A}_{\Lambda}\right), E\right) \geq(2 n+1) \frac{1}{2} \mu l^{\beta-1}$. Thus by the resolvent decay estimate, see Theorem B.1, we find

$$
\left\|\chi^{\text {int }}\left(H_{\Lambda}(A)-E\right)^{-1} \chi^{\text {out }}\right\| \leq \frac{2 c_{1}}{\mu(2 n+1)} l^{1-\beta} \exp \left(-c_{2}\left(\frac{1}{2}(2 n+1) \mu l^{\beta-1}\right)^{1 / 2} l / 4\right),
$$

for $l \geq 4$. On the other hand if $\Lambda$ is $\left(E, b_{0} C_{\infty}, \alpha\right)$-balanced, then

$$
\left\|\chi^{\text {out }}\left(H_{\Lambda}(A)-E\right)^{-1} \chi^{\text {in }}\right\| \leq\left(1+b_{0} C_{\infty} l^{\alpha}\right) \mid \text { r.h.s. of }(5.23) \mid \text {. }
$$

Thus we conclude from (5.23) and (5.24) that there exists an $\hat{L}_{G}=\hat{L}_{G}(\tau, \xi, \mu, n, \alpha, \kappa)$, such that $\Lambda$ is $(\gamma, E)$-good provided $(5.22)$ holds, $\Lambda$ is $\left(E, b_{0} C_{\infty}, \alpha\right)$-balanced, and

$$
l \geq \hat{L}_{G} b_{0}^{\kappa} .
$$

It remains to estimate the probability. Let $\alpha>3 C_{1}\left(\kappa^{-1}+\rho\right)$ (with $C_{1}$ as in Theorem 5.3) and let $l$ satisfy (5.25). Then using the conclusion of the sentence leading up to (5.25), we find

$$
\begin{aligned}
\mathbb{P}\{\forall & \left.\in I, \Lambda_{l}(x) \text { or } \Lambda_{l}(y) \text { is }(\gamma, E)-\operatorname{good}\right\} \\
& \left.\geq \mathbb{P}\left\{\forall E \in I, \Lambda_{l}(x) \text { or } \Lambda_{l}(y) \text { is }\left(E, b_{0} C_{\infty}, \alpha\right)\right)-\text { balanced and }(5.22) \text { holds for } \Lambda_{l}(x) \text { and } \Lambda_{l}(y)\right\} \\
& \left.\geq 1-\mathbb{P}\left\{\operatorname{not}\left[\forall E \in I, \Lambda_{l}(x) \text { or } \Lambda_{l}(y) \text { is }\left(E, b_{0} C_{\infty}, \alpha\right)\right)-\text { balanced }\right]\right\} \\
& -\mathbb{P}\left\{\operatorname{not}\left[(5.22) \text { holds for } \Lambda_{l}(x) \text { and } \Lambda_{l}(y)\right]\right\} \\
& \geq p_{l, x} p_{l, y}-p_{1},
\end{aligned}
$$


where we set

$$
\left.p_{1}:=\mathbb{P}\left\{\operatorname{not}\left[\forall E \in I, \Lambda_{l}(x) \text { or } \Lambda_{l}(y) \text { is }\left(E, b_{0} C_{\infty}, \alpha\right)\right)-\text { balanced }\right]\right\}
$$

and we used that by independence

$$
\mathbb{P}\left\{\operatorname{not}\left[(5.22) \text { holds for } \Lambda_{l}(x) \text { and } \Lambda_{l}(y)\right]\right\}=1-p_{l, x} p_{l, y}
$$

where

$$
p_{l, x}=p_{l, y}=p_{l, z}:=\mathbb{P}\left\{(5.22) \text { holds for } \Lambda_{l}(z)\right\} .
$$

It remains to estimate $(5.27)$. Observe that by Corollary 5.4 with $(\xi \rightarrow 2 \xi)$ there exists an $l_{\text {initial }}=$ $l_{\text {initial }}\left(\tau, \xi, c_{u}, c_{v}, c_{\delta}\right)$ such that for all $l \geq l_{\text {initial }}$

$$
p_{l, x} \geq 1-\frac{1}{4} l^{-2 \xi} .
$$

On the other hand by Lemma 5.2

$$
p_{1} \leq 3 C_{0}^{2} \mu^{-3} l^{-\alpha / 3} \leq \frac{1}{4} l^{-2 \xi}
$$

where we used that the width of the interval for which we want to prove localization is bounded by $\mu$, and the last inequality follows if we choose $\alpha$ sufficiently large. Thus inserting (5.30) and (5.29) into (5.27), we find that (5.26) is bonded from below by

$$
1-\frac{1}{4} l^{-2 \xi}-\frac{1}{2} l^{-2 \xi} \geq 1-l^{-2 \xi}
$$

for $l \geq l_{\text {initial }}$ satisfying $(5.25)$.

For $\Theta>0$, and $q>0$ we introduce the following hypothesis.

$W(I, l, \Theta, q): \quad$ For all $E \in I$ and $\Lambda=\Lambda_{l}(x), x \in \mathbb{Z}^{2}$, the following estimate holds:

$$
\mathbb{P}\left\{\operatorname{dist}\left(\sigma\left(H_{\Lambda}(A)\right), E\right) \leq \exp \left(-l^{\Theta}\right)\right\} \leq l^{-q}
$$

The following Lemma is a consequence of Theorem 5.1.

Lemma 5.7 Suppose the assumptions of Theorem 5.1 hold. Let $\Theta>0, q>0$, and $0<\kappa \leq 1$. Let $I \subset \mathbb{R}$ be a finite interval with $\inf I \geq b_{0} / 2$. Then there exists a constant $l_{W}^{*}=l_{W}^{*}\left(I, \Theta, q, K_{0}, K_{1}, \delta, \mu, \kappa, \rho\right)$ such that $W(I, l, \Theta, q)$ holds for all $l \geq l_{W}^{*} b_{0}^{\kappa}$.

Proof of Theorem 2.5. We consider only the upper band edges. The lower band edges are proven analogously. Fix $\xi \in(0, \tau-2)$ and let $\beta=\frac{1}{2}\left(1-\frac{\xi+2}{\tau}\right)$. Choose, $0<\Theta<\beta / 2$ and $q>2$ and set $\kappa:=\frac{1}{2} \min \left((2-2 \beta)^{-1}, 1\right)$. By Lemma 5.6 there exists an $l_{G}=l_{G}\left(\tau, \xi, c_{u}, c_{v}, c_{\delta}, K_{0}, K_{1}, \delta, \mu, \rho\right)$ such that $G\left(I_{l}, l, \gamma_{l}, \xi\right)$ holds with $I_{l}=I_{n}^{+}\left[B_{\omega_{+}}\right]+\left[-\frac{1}{2} \mu l^{\beta-1}, 0\right]$ and $\gamma_{l}:=l^{\beta-1}$ for all $l \geq l_{G} b_{0}^{\kappa}$. By Lemma 5.7 there exists an $l_{W}^{*}$ (depending on $\left.\Theta, q, K_{0}, K_{1}, \delta, \mu, \kappa, \rho\right)$ such that $W\left(I_{l}, l, \Theta, q\right)$ is satisfied for $l \geq l_{W}^{*} b_{0}^{\kappa}$ and thus also for $l \geq l_{0}:=\max \left(l_{W}^{*} b_{0}^{\kappa}, l_{G} b_{0}^{\kappa}\right)$. Thus one now apply the multiscale analysis as outlined in [14] for the interval $J_{0}:=I_{l_{0}}$ (This is explained in detail in [5]).

Having established the application of the multiscale analysis we can now show using standard arguments that $H\left(A_{\omega}\right)$ has pure point spectrum in $J_{0}$ for almost all $\omega \in \Omega$. We write $J_{0}=I_{n}^{+}\left[B_{\omega_{+}}\right]+\left[-e_{0}, 0\right]$, with $e_{0}:=\frac{1}{2} \mu l_{0}^{\beta-1}$. Observe that by Theorem 3.2 there is no spectrum in the interval $\left(I_{n}^{+}\left[B_{\omega_{+}}\right], I_{n+1}^{-}\left[B_{\omega_{-}}\right]\right)$. Thus to conclude that an interval of the form (2.22) contains pure point spectrum, it suffices to show that $E_{n}^{+} \in J_{0}$ and an analogous statement for the corresponding lower band edge. To this end note that we have

$$
C_{n}\left(K_{2}^{ \pm} b_{0}^{-1 / 2}+K_{3}^{ \pm} b_{0}^{-2}\right) \leq \frac{1}{2} e_{0}=\frac{1}{4} \mu b_{0}^{\kappa(\beta-1)}\left[\max \left(l_{W}^{*}, l_{G}\right)\right]^{\beta-1},
$$


if $b_{0}$ is sufficiently large, noting that $b_{0}^{\kappa(\beta-1)} \geq b_{0}^{-1 / 4}$ by the choice of $\kappa$. Since $K_{3}^{ \pm} \leq\left[K_{2}^{ \pm}\right]^{2}$, the threshold for $b_{0}$ grows with $\left[K_{2}^{ \pm}\right]^{4}$ for large $K_{2}^{ \pm}$. By (5.31) and the definition (5.14) (recall (3.2)) it now follows that $E_{n}^{+} \in J_{0}$. In view of Theorem 2.2 an analogous argument now implies (2.23).

\section{A Analytic properties of the resolvent $R(z)$}

We cite the following Lemma found in [12].

Lemma A.1 For any $z \in \mathbb{C} \backslash \sigma(H)$, the integral kernel of the resolvent $R(z)=(H-z)^{-1}$ of the magnetic Hamiltonian $H=\left(p_{1}+\frac{B_{0}}{2} x_{2}\right)^{2}+\left(p_{2}-\frac{B_{0}}{2} x_{1}\right)^{2}$, with constant magnetic field $B_{0}$, can be expressed in terms of the $\Gamma(\cdot)$-function and confluent hypergeometric function $U(\cdot, \cdot \cdot \cdot)$ as follows:

$$
R(z)(x, y)=\frac{1}{4 \pi} \Gamma(w) U(w, 1 ; \zeta) \exp \left(-\frac{1}{2} \zeta-i \frac{B_{0}}{2}[x, y]\right)
$$

with $[x, y]=x_{1} y_{2}-x_{2} y_{1}, w=\frac{1}{2}-\frac{z}{2 B_{0}}$, and $\zeta=\frac{B_{0}}{2}|x-y|^{2}$.

Fix $n \in \mathbb{N}_{0}$. Suppose $z \in \mathbb{C}$ is in the (3.16) neighborhood of the $n$-th Landau level. In terms of $w$ this is equivalent to

$$
-n-\frac{1}{2} \leq \operatorname{Re} w \leq-n+\frac{1}{2}, \quad|\operatorname{Im} w| \leq 1 / 2 .
$$

It is well known that the $\Gamma$ function is a meromorphic function with simple poles at $0,-1, \ldots$. Thus

$$
\left|\Gamma\left(\frac{1}{2}-\frac{z}{2 B_{0}}\right)\right| \leq C_{n}\left[\frac{B_{0}}{\left|z-B_{0}(2 n+1)\right|}+1\right],
$$

for some constant $C$ (depending on $n$ ). Furthermore, there exists a constant $C$ (depending on $n$ ) such that

$$
\begin{aligned}
U(w, 1 ; \zeta) & \leq C\left(1+\ln \zeta+\zeta^{n+1}\right), \\
U_{3}(w, 1 ; \zeta) & \leq C\left(1+\zeta^{-1}+\zeta^{n+1}\right),
\end{aligned}
$$

for all $\zeta>0$. This estimate can be seen as follows. We use the the integral representation 13.2.5. in [1],

$$
\Gamma(a) U(a, 1 ; \zeta)=\int_{0}^{\infty} e^{-\zeta t} t^{a-1}(1+t)^{-a} d t, \quad \operatorname{Re} a>0, \quad \operatorname{Re} \zeta>0 .
$$

Note that (A.6) can a priori only be applied if Rea $>0$, but we need it for $w$ satisfying (A.2). To circumvent this problem, we iterate the recurrence relation 13.4.15. in [1]

$$
U(a, 1 ; \zeta)=(1+2 a+\zeta) U(a+1,1 ; \zeta)-(a+1)^{2} U(a+2,1 ; \zeta),
$$

$n+1$ times, which yields

$$
U(w, 1 ; \zeta)=P_{n}(a, \zeta) U(w+n+1,1 ; \zeta)+Q_{n}(w, \zeta) U(w+n+2,1 ; \zeta),
$$

for some polynomials $P_{n}$ and $Q_{n}$ with degree at most $n+1$ in $\zeta$ and $2(n+1)$ in $w$. Now (A.4) and (A.5) can be shown using (A.6) to estimate the right hand side of (A.7) (observe that the $\Gamma$ function has no zeros in the set $\left[\frac{1}{2}, \frac{5}{2}\right]+i\left[-\frac{1}{2}, \frac{1}{2}\right]$ ). The large $\zeta>0$ behavior in (A.4) is now trivial to see. For the small $\zeta$ behavior note that (A.6) diverges logarithmically in $\zeta$ as $\zeta \downarrow 0$, and that its derivative with respect to $\zeta$ diverges like $\zeta^{-1}$. 


\section{B Combes-Thomas decay estimate}

Define the function $\rho(x)=\left(1+|x|^{2}\right)^{1 / 2}$. Let $\widetilde{H}$ be an operator of the form $H_{\Lambda}(A)$. Define

$$
\widetilde{H}(\alpha):=e^{i \alpha \rho} \widetilde{H} e^{-i \alpha \rho}=\widetilde{H}-\alpha \nabla \rho \cdot(-i \nabla-a)-(-i \nabla-a) \cdot \alpha \nabla \rho+\alpha^{2}|\nabla \rho|^{2} .
$$

Since $|\nabla \rho|$ and $|\Delta \rho|$ are bounded and $(-i \nabla-a)$ is infinitesimally small with respect to $\widetilde{H}$, we obtain that $\widetilde{H}(\alpha)$ is an analytic family of type A on $\mathbb{C}$. Using this property one can show the following result, following the proof of Theorem 2.4.1. in [14].

Theorem B.1 Let $R>0$. Then there exists a $c_{1}=c_{1}(R)$ and $c_{2}=c_{2}(R)$ such that the following holds. $(r, s) \subset \rho(H) \cap(-R, R), E \in(r, s)$ and $\eta=\operatorname{dist}\left(E,(r, s)^{c}\right)>0$ imply the estimate

$$
\left\|\mathbf{1}_{B}(H(A)-E)^{-1} \mathbf{1}_{D}\right\| \leq c_{1} \eta^{-1} \exp \left[-c_{2}(s-r)^{1 / 2} \eta^{1 / 2} \delta\right]
$$

where $B$ and $D$ denote a set in $\mathbb{R}^{2}$ and $\operatorname{dist}(B, D)=: \delta>0$.

\section{References}

[1] Abramowitz, M., Stegun, I.: Handbook of mathematical functions with formulas, graphs, and mathematical tables. National Bureau of Standards Applied Mathematics Series, U.S. Government Printing Office, Washington, D.C. 1964.

[2] Combes, J.M., Hislop, P.: Landau Hamiltonians with random potentials: localization and the density of states. Commun. Math. Phys. 177, 603-629 (1996)

[3] Dorlas, T.C., Macris, N., Pulé, J.V.: Localisation in a single-band approximation to random Schrödinger operators in a magnetic field. Helv. Phys. Acta 68, 329-364 (1995)

[4] Von Dreifus, H., Klein, A.: Localization for random Schrödinger operators with correlated potentials. Commun. Math. Phys. 140, 133-147 (1991)

[5] Erdős, L., Hasler, D.: Wegner estimate and Anderson localization for random magnetic fields. Preprint. arXiv: 1012.5185

[6] Erdős, L., Hasler, D.: Anderson localization for random magnetic Laplacian on $\mathbb{Z}^{2}$. Preprint. arXiv:1101.2139

[7] Fischer, W., Leschke, H., Müller, P.: Spectral localization by Gaussian random potentials in multidimensional continuous space. J. Statis. Phys. 101, 935-985 (2000)

[8] Ghribi, F., Hislop, P.D., Klopp, F., Localization for Schrödinger operators with random vector potentials. Adventures in mathematical physics, 123-138, Contemp. Math., 447, Amer. Math. Soc., Providence, RI, 2007.

[9] Germinet, F. and Klein, A: Explicit finite volume criteria for localization in continuous random media and applications. Geom. Funct. Anal. 13, 1201-1238 (2003)

[10] Hislop, P.D., Klopp, F.: The integrated density of states for some random operators with non-sign definite potentials. J. Funct. Anal. 195, 12-47 (2002)

[11] Kirsch W., Stollmann P., Stolz G.: Anderson localization for random Schrödinger operators with long range interactions. Commun. Math. Phys. 195 (1998), no. 3, 495-507.

[12] Korotyaev E., Pushnitski A.: A trace formula and high-energy spectral asymptotics for the perturbed Landau Hamiltonian. J. Funct. Anal., 217, 221-248, (2004). 
[13] Klopp, F., Nakamura, S., Nakano, F., Nomura, Y.: Anderson localization for $2 D$ discrete Schrödinger operators with random magnetic fields. Ann. Henri Poincaré 4 795-811 (2003)

[14] Stollmann, P.: Caught by Disorder, Bound States in Random Media, Birkhäuser, Boston, 2001

[15] Ueki, N.: Wegner estimates and localization for random magnetic fields. Osaka J. Math. 45, 565-608 (2008)

[16] Wang, W.-M.: Microlocalization, percolation and Anderson localization for the magnetic Schrödinger operator with a random potential. J. Funct. Anal. 146 1-26 (1997) 